\title{
Natural Frequency of Tension Cable Based on Moment Inertia to Span Ratio: Determination Using Analytical, Numerical and Experimental Study
}

\author{
Guntur Nugroho ${ }^{1^{*}}$ \\ ${ }^{1}$ Department of Civil Engineering, Faculty of Engineering, \\ Universitas Muhammadiyah Yogyakarta, Yogyakarta 55183, INDONESIA \\ *Corresponding Author
}

DOI: https://doi.org/10.30880/ijscet.2020.11.02.010

Received 30 July 2020; Accepted 30 August 2020; Available online 02 September 2020

\begin{abstract}
Health monitoring using vibration technique is usually conducted on cable structure. The hanger cable on the suspension bridge has a difference of span. To predict axial force of cable, the beam-string theory includes a parameter of bending stiffness. However, string theory has neglected the effect of bending stiffness. The shorter the span of the cable the greater the effect of the bending stiffness would be. This paper raises parameter moment of inertia to span ratio (I/L) to determine the apropriate analytical formula between string and beam-string. Experimental research was conducted using a vibration technique. The specimens use solid cylindrical steel beam, having length specimens of $2 \mathrm{~m}$, hinge-hinge of boundary condition, and difference variations $\mathrm{I} / \mathrm{L}$ of $0.024,0.08$, $0.58,1.53$ and 10.22. Numerical analysis was simulated by using Abaqus software v 6.13. The result shows that the ratio of I/L equally lowers than 0.082 has close to the analytical string theory. The ratio of $\mathrm{I} / \mathrm{L}$ greather than 0.082 has close to the beam string theory.
\end{abstract}

Keywords: Health monitoring, force prediction, string theory, beam-string theory, moment of inertia

\section{Introduction}

Vibration method has been widely applied to estimate cable force in bridge engineering since because of simplicity and speediness as presented in Nugroho, Priyosulistyo, and Suhendro (2014). It was applied on tensioned cable structure such as of suspension bridge as well as of cable-stayed bridge. On the suspended cable structure, such as hanger-on a suspension bridge, a vibration method is the most common approach used for the force measurement of hanger cables. Evaluation of force of hanger cables is very important to ensure the stability of the structure.

Recently, vibration methods are not only applied to evaluate the force of the cable but also to develop the analitical formula based on the fundamental theory of string as well as theory of beam. A formula that considers sagextensibility, derived from the modern cable theory, was proposed by Russell and Lardner (1998). This method requires unstrained length of the cable as well as the solution of a nonlinear frequency equation using trial-and-error. This method was limited by the unavailable data of unstrained length in practice.

Zui et al. (1998) solved the limitation of the Russell and Lardner (1998) by proposing a practical formulas for the vibration method was proposed by taking into account the effects of flexural rigidity and sag of a cable. This method was not limited by the unavailable data of unstrained length in practice. The approximate solutions with high accuracy for the equation of inclined cable with flexural rigidity was used to consider in these pactical formulation. The force of 
cable can be calculated by using the natural frequencies of low-order modes. The accuracy of the proposed practical formulas was verified not only using the comparison of the values of practical formulas with experimental values for short and middle length cables but also calculated values by using finite element method for very long cables.

Tabatabai, Mehrabi and Yen (2003) also solved the limitation of the Russell and Lardner (1998), by proposing a nondimensional equation that based on parametric studies using a unified finite-difference formulation. This method was not limited by the unavailable data of unstrained length in practice. This method raises parametric study that not only consider the effect of bending stiffnes of cable and its sag-extensibility, but also provides a tool for accurate detemination of vibration mode shapes and natural frequencies. The proposed formulation was verified by using available theoretical solutions and compared by using finite-element analysis. The accuracy of vibration method formulas proposed by Russell and Lardner (1998), Zui et al (1998) and Tabatabai et al (2003) were verified. However, these formulation were expressed either in a transcendental equation (Tabatabai et al., 2003), or in a complex piecewise function (Zui et al. 1998). A trial-and-error calculation should be conducted to estimate the cable tension.

Fang and Wang (2012) solved the limitation of the Zui et al (1998) and Tabatabai et al (2003) by proposing non dimensional parameter that eliminated trial-and-error calculation in estimating the cable tension. The non dimensional parameter was included on the formulation of beam theory. Experimental was conducted using specimen of $3.4 \mathrm{~m}$ long cable with negligibly small sag effect, the results shows that the Fang and Wang (2012) and the Zui et al (1998) have the maximum errors are $0.8 \%$ and $1.1 \%$, respectively.

Aplication of the vibration method used for field measurement of cable force under the construction of cable system bridges such as an arch bridge stiffened with suspended cables or suspension bridge as well as cable-stayed bridge. Kim and Park (2007) presents a comparative study of field measurment of cable supported bridge using vibration-based tension estimation techniques. Force measurement was conducted on the four inclined stay-cables on the Seohae Grand Bridge by using various formula of string theory, beam theory (Zui et al., 1998). The error was verified by using the lift-of method. The result show that the error of string theory, beam theory (Zui et al., 1998) have $0,9 \%, 1,3 \%$ and $3,2 \%$ respectively.

Soojin, et al. (2013) had been conducted the fielt test of force measurement of cable by using various tension measurement methods. The under constructio of cable stayed bridge (Hwamyung Bridge in Korea) was elected. Force measurement of cable had been conducted by using three widely used methods that is the lift-off test, electromagnetic sensor method, and vibration method. The result shows that the lift-off test, electromagnetic sensor method, and vibration method have less than $3.5 \%$ difference from the design tensions.

Although the vibration method have several formula, it was based on the fundamental theory of string as well as beam theory. The different between the string theory and beam theory are the parameter of bending stifness. The string theory neglects the parameter effect of bending stifnes but the beam theory includs the parameter of bending stifness. Application of cable structure in suspension bridge as well as of cable stayed bridge has significant difference of span. The shorter the span of the cable the closer the behavior cable to the beam theory would be. The longer the span of the cable the closer the compatible to the string theory would be. The objective of this paper is to introduce the new parameter of $\mathrm{I} / \mathrm{L}$ to determine the cable, wether will apropriate to the beam or the string theory.

\section{Analytical theory}

\subsection{The string theory}

The first is string theory that ignores the effect bending stiffness. The governing equation of motion related to the displacement for a continuously vibrating cable can be written as following (Zui et al.,1998), (Saxon and Chan, 1953), (Irvine, 1981):

$$
T \frac{\partial^{2} w}{\partial x^{2}}-m \frac{\partial^{2} w}{\partial t^{2}}+h \frac{\partial^{2} w}{\partial x^{2}}-E I \frac{\partial^{4} w}{\partial t^{4}}=0
$$

For thin taut cables, the most basic of vibrating dynamic cables, neglecting the flexural stiffness of the cable, additional tension due to the cable vibration, and sag, the equation of motion (1) reduces to:

$$
T \frac{\partial^{2} w}{\partial x^{2}}=m \frac{\partial^{2} w}{\partial t^{2}}
$$

Equation (2) is the fundamental partial differential equation for a thin taut vibrating cable and can be extended too many different applications. The equation is simplified to relating the cable's tension to the linear mass of the system by way of the partial differential equation. The solution to this equation, found in Dynamics of Structures (Clough and Penzien, 1995), can be used to estimate the tension of a cable through the frequency of vibration. Solving the eigenvalue problem associated with (2) yields the equation for the natural frequencies of the cable: 


$$
f_{n}=\frac{n}{2 l} \sqrt{\frac{T}{m}}
$$

By solving equation 3 for the tension yields, the cable force (T) can be written as (Nugroho et al., 2014), (Fang, and Wang, 2012), (Kim and Park, 2007):

$$
T=4 m L^{2}\left(\frac{f_{n}}{n}\right)^{2}
$$

where $\mathrm{f}_{\mathrm{n}}$ denotes the nth natural frequency in $\mathrm{Hz}$; and $\mathrm{T}, \mathrm{m}$, and $l$ denote the tension, mass density, and length, respectively.

\subsection{The beam string theory}

Axial forces, performing in a flexural direction on beam element (Fig.1), may also have a considerable affect at the vibration behavior of the member, generally resulting in changes of frequencies as well as mode shapes (Irvine, 1981) [9]. When considering free vibrations of a prismatic member having uniform physical properties, the equation of motion, including the effect of axial force, $\mathrm{T}$ overall its length, as follows:

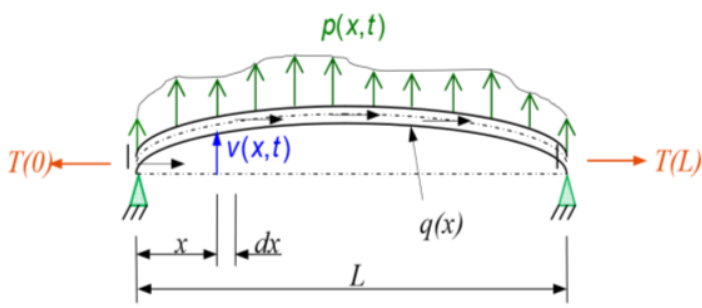

Fig. 1 - Tranverse vibration of beam (Irvine, 1981).

$$
\begin{aligned}
& E I \frac{\partial^{4} V(x, t)}{\partial t^{4}}-T \frac{\partial^{2} V(x, t)}{\partial x^{2}}+m \frac{\partial^{2} V(x, t)}{\partial t^{2}}=0 \\
& \frac{\phi^{i V}(x)}{\phi(x)}-\frac{T \phi^{\prime \prime}(x)}{E I \phi(x)}=-\frac{m \ddot{Y}(t)}{E I Y(t)}=\alpha^{4}
\end{aligned}
$$

the two independent ordinary differential equations are obtained as given by

$$
\begin{aligned}
& \ddot{Y}(t)+\omega^{2} Y(t)=0 \\
& \phi^{i V}(x)+g^{2} \phi^{\prime \prime}(x)-\alpha \phi(x)=0
\end{aligned}
$$

in which $\omega^{2}$ is defined by

$$
\omega^{2}=\frac{\alpha^{4} E I}{\bar{m}}
$$

and $\mathrm{g}^{2}$ is given by

$$
g^{2}=\frac{T}{E I}
$$

The time-dependent Eq. 7 show that a uniformly distributed axial force does not affect the simple harmonic character of the free vibration; however, it does affect the mode shapes and frequencies due to the presence of the term $-\mathrm{g} 2 \varphi$ ”(x) in Eq. 8. The solution of Eq. 7 yields the general form:

$$
\phi(x)=G e^{s x}
$$


Introducing Eq. 10 (3.24) into Eq. 8 (3.22b) and dividing by . $A e^{\lambda x}$, it can be concluded that the exponent s must satisfy the algebraic equation:

$$
S^{4}-g^{2} S^{2}-\alpha^{4}=0
$$

$$
\text { (i.e.EIS } \left.S^{4}-T S^{2}-\omega^{2} m=0\right)
$$

whose roots are (Clough and Penzien, 1995)

$$
\begin{aligned}
& S_{1,2}= \pm \sqrt{\frac{T}{2 E I}\left(\sqrt{1+\frac{4 \omega^{2} \bar{m} E I}{T^{2}}+1}\right)} \\
& S_{3,4}= \pm \sqrt{\frac{T}{2 E I}\left(\sqrt{1+\frac{4 \omega^{2} \bar{m} E I}{T^{2}}-1}\right)}
\end{aligned}
$$

so that the general solution of Eq. 8 (3.22b) is expressed in the form

$$
\phi(x)=C_{1} e^{S 1 x}+C_{2} e^{S 2 x}+C_{3} e^{S 3 x}+C_{4} e^{S 4 x}
$$

where $\mathrm{C} 1, \mathrm{C} 2, \mathrm{C} 3$ and $\mathrm{C} 4$ are coefficients which is determined from the boundary conditions. Since the pairs of roots s1, s2 and s3, s4, are opposite each other and are, respectively, real and purely imaginary

$$
S_{1,2}= \pm \varepsilon, S_{3,4}= \pm i \delta
$$

where $\mathrm{l}$ is the imaginary unit. Expressing the exponential functions in terms of their trigonometric and hyperbolic equivalents and setting the entire imaginary part to zero, Eq. 15 can be re-written in the form

$$
\phi(x)=D_{1} \cos \delta x+D_{2} \sin \delta x+D_{3} \cosh \varepsilon x+D_{4} \sinh \varepsilon x
$$

in which $\delta=\mathrm{ts} 3$ and $\varepsilon=\mathrm{s} 1$

$$
\begin{aligned}
& \delta=\sqrt{\frac{T}{2 E I}\left(\sqrt{1+\frac{4 \omega^{2} \bar{m} E I}{T^{2}}}-1\right)} \\
& \delta=\sqrt{\frac{T}{2 E I}\left(\sqrt{1+\frac{4 \omega^{2} \bar{m} E I}{T^{2}}}+1\right)}
\end{aligned}
$$

The coefficients D1, D2, D3, and D4 can be evaluated by exactly the same procedure presented for the system without axial force. Considering the simply-supported tie-rod with the uniform section, subjected to a constant axial tensile force, its four boundary conditions are the same as follows

$$
\begin{aligned}
& \phi(x)=D_{1} \cos 0+D_{2} \sin 0+D_{3} \cosh 0+D_{4} \sinh 0 \\
& \phi(x)=-\delta^{2} D_{1} \cos 0-\delta^{2} D_{2} \sin 0-\varepsilon^{2} D_{3} \cosh 0 \\
& -\varepsilon^{2} D_{4} \sinh 0
\end{aligned}
$$

These two equations give $\mathrm{D} 1=\mathrm{D} 3=0$

$$
\phi(L)=D_{2} \sin \delta L-D_{4} \sinh \varepsilon L \phi(x)=-\delta^{2} D_{2} \sin \delta L-\varepsilon^{2} D_{4} \sinh \varepsilon L
$$


Adding these two equations and replacing D4 with an expression of D2. Only D2 remains as a nonzero constant

$$
\phi(L)=D_{1} \sin \delta x\left(1+\frac{\delta^{2}}{\varepsilon^{2}}\right)
$$

Excluding the trivial solution $\mathrm{D} 2=0$, boundary condition $\varphi(\mathrm{L})=0$ can be satisfied only when $\sin \delta L=0, \delta=\pi / L$ and $\mathrm{n}=1,2,3$.

The frequency can be expressied as following

$$
\begin{aligned}
& \omega_{n}=n \pi \sqrt{\frac{n^{2} \pi^{2} E I}{m L^{4}}+\frac{T}{m L^{4}}} \mathrm{Rad} \\
& f_{n}=\frac{n}{2 L} \sqrt{\frac{n^{2} \pi^{2} E I}{m L^{4}}+\frac{T}{m L^{4}}} \mathrm{~Hz}
\end{aligned}
$$

\section{Numerical model}

Numerical moodelling was simulated using Abaqus software. The hanger was modeled using solid element. By using a solid cylindrical beam, the the cross section of the specimen has similar span and the diference of diameter as presented in Table 1. The hanger was modelled using simplifiying and some assumptions as follows.

Table 1 - Specimen of hanger using diference parameter of $\mathrm{I} / \mathrm{L}$

\begin{tabular}{cccccc}
\hline No & Diameter $(\mathbf{m m})$ & Length $\mathbf{L}(\mathbf{m m})$ & Momen of Inertia I $\left(\mathbf{m m}^{\mathbf{4}}\right)$ & Elastic modulus $(\mathbf{G P a})$ & $\mathbf{I} / \mathbf{L}$ \\
\hline 1 & 5.6 & 2000 & 48.27 & 190.4 & 0.024 \\
2 & 7.6 & 2000 & 163.77 & 196.5 & 0.082 \\
3 & 12.4 & 2000 & 1160.53 & 196.6 & 0.580 \\
4 & 15.8 & 2000 & 3059.13 & 196,6 & 1.530 \\
5 & 25.4 & 2000 & 20431.71 & 199,7 & 10.21 \\
\hline
\end{tabular}

\subsection{Material and Section properties}

The material is homogeneous, isotropic and linearly elastic. Steel material has material properties of poison ratio and mass density of 0.3 and $78.10^{-6} \mathrm{~N} / \mathrm{mm}^{3}$ respectively. The specimen has the elastic modulus, dimension and length as presented in Table 1 .

\subsection{Load}

The axial load $(\mathrm{T})$ was simulated as a pressure $(\mathrm{P}=\mathrm{T} / \mathrm{A})$ uniformly distributed on the center of hanger element. The axial tensile force of $4000 \mathrm{~N}$ was equally applied to the specimens. By implementing the pressure load using a negative value, it will be defined as a tension force.

\subsection{Boundary Condition}

The end of the specimen has a two different shape. The flat side end used for the application of axial tension loading was shown in Fig 2 while the other end uses the mounting shape as shown in Fig 3. The flat end shape shown in Fig 2 was used to apply the axial tension force of the specimen. The boundary condition of the end shape of the specimen in Fig 2 is allowed to rotate and to deflect in the axis direction. On the other side, the boundary condition of hinge-hinge was implemented by using the mounting shape of the end, and it is constrained as hinge boundary condition as shown in Fig 3. 


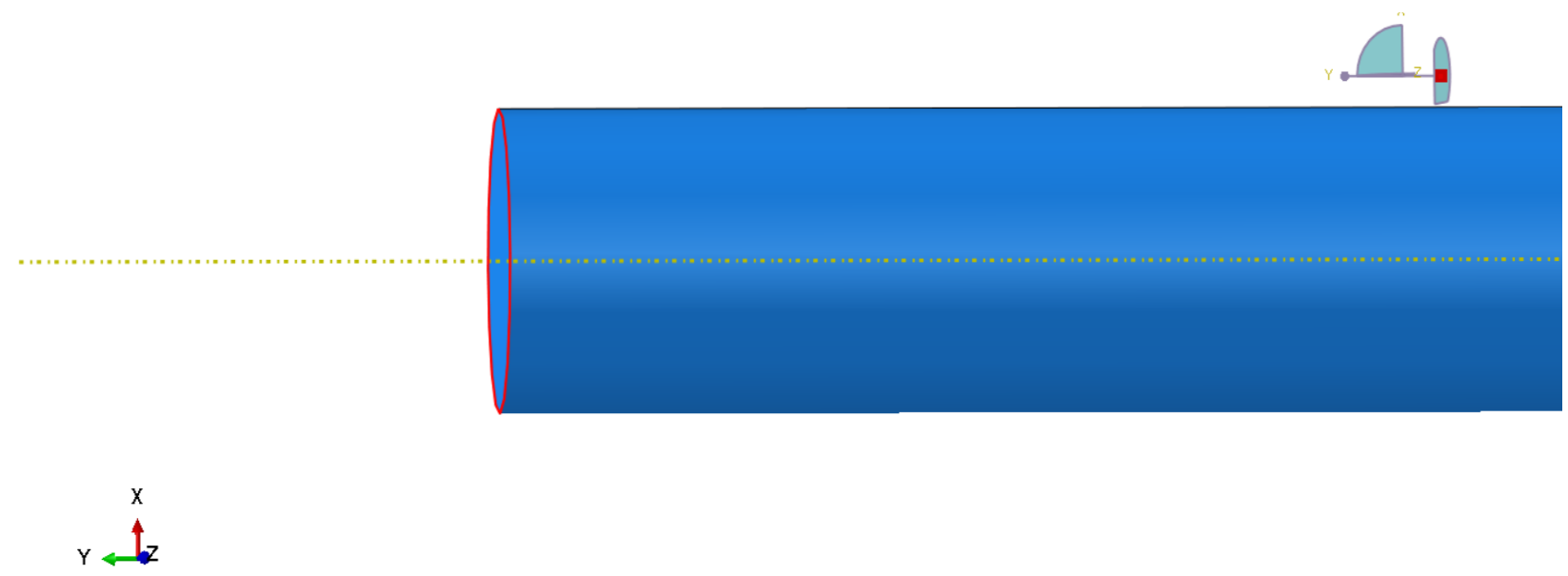

Fig. 2 - Flate end shape pf boundary condition

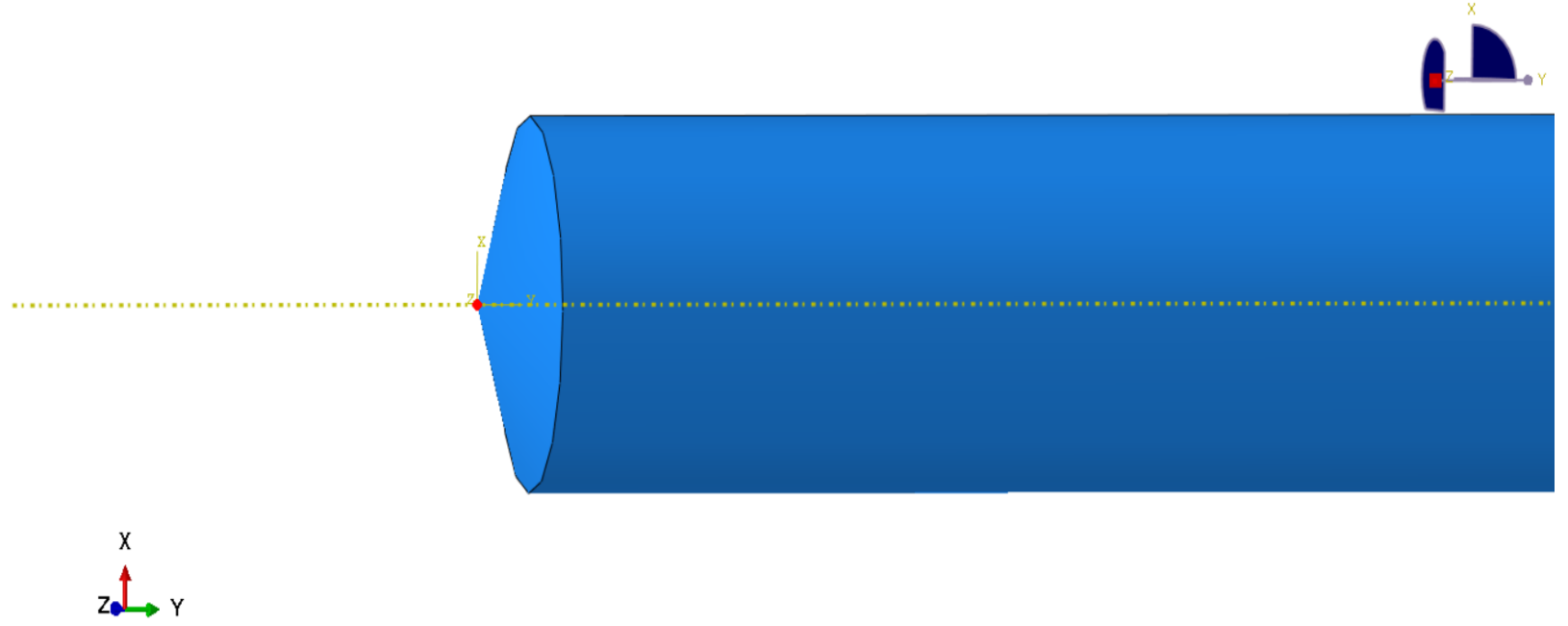

Fig. 3 - Mounting end shape of boundary condition

\subsection{Modal Frequency}

To find the modal frequency of the model, the step in abaqus consists of two step as follows. To find the modal frequency of the model, the step in abaqus consists of two step as follows.

Step 1: Axial tension force is applied on the top end using pressure load. It will affect to the internal stress in the model and will be followed by increasing of tangent stiffness. The nonlinear geometry analysis must be performed in this step. Step 2: After the hanger model has been tensioned and elongated in step 1. The eigenvalue analysis is performed to obtain the modal frequencies of the model using the mass matrix and the tangent stiffness matrix. The mode shape of the natural frequency visualises in Fig. 4 - Fig. 6.

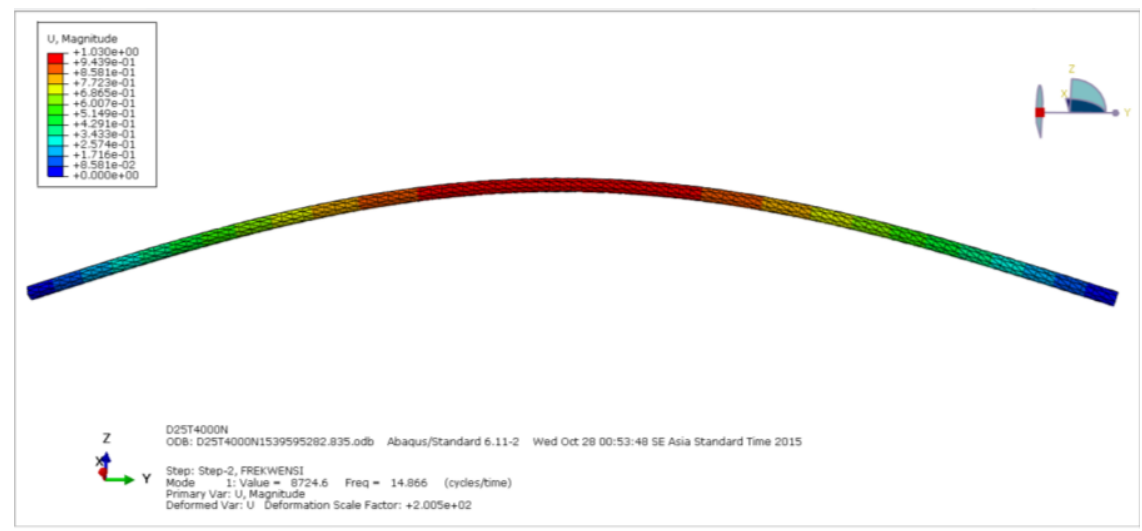

Fig. 4 - The first mode of visualisation 


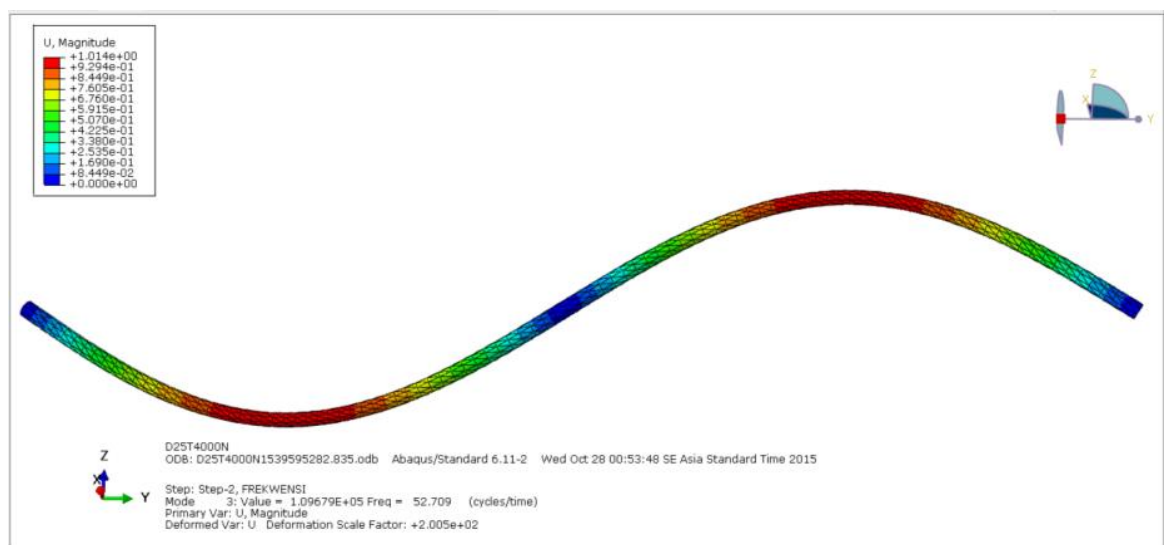

Fig. 5 - The second mode of visualisation

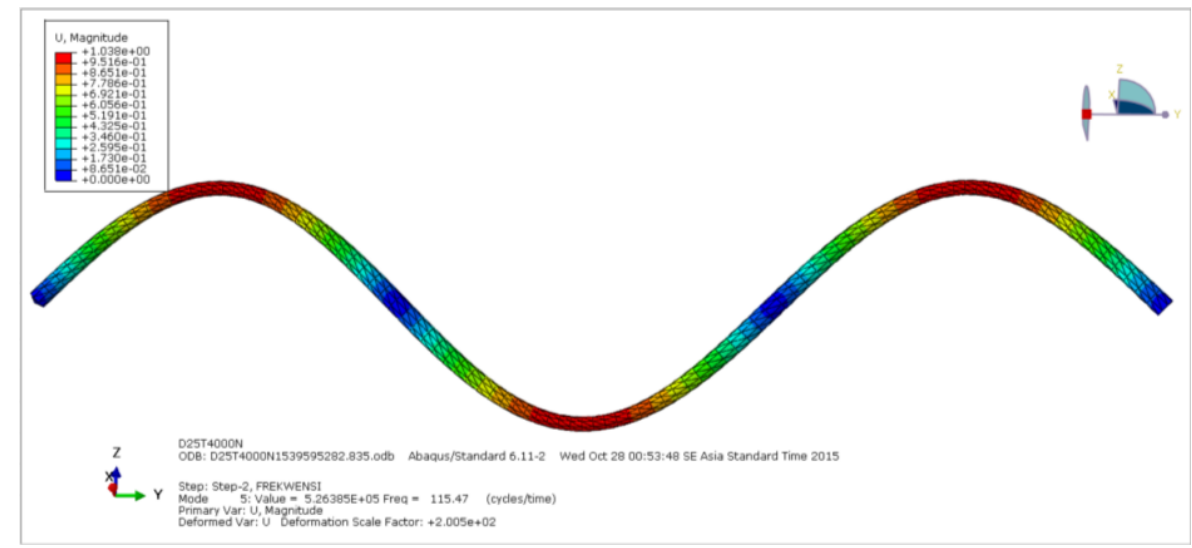

Fig. 6 - The third mode of visualisation

\section{Experimental Method}

A laboratory experiment was conducted using a solid circular steel specimen. The specimen uses several variations of the moment of inertia to span ratio $\mathrm{I} / \mathrm{L}$ which is similar to the numerical modeling $(0.024,0.08,0.58,1.53$ and 10.22). The test set up of the experiment was shown in Fig 7.

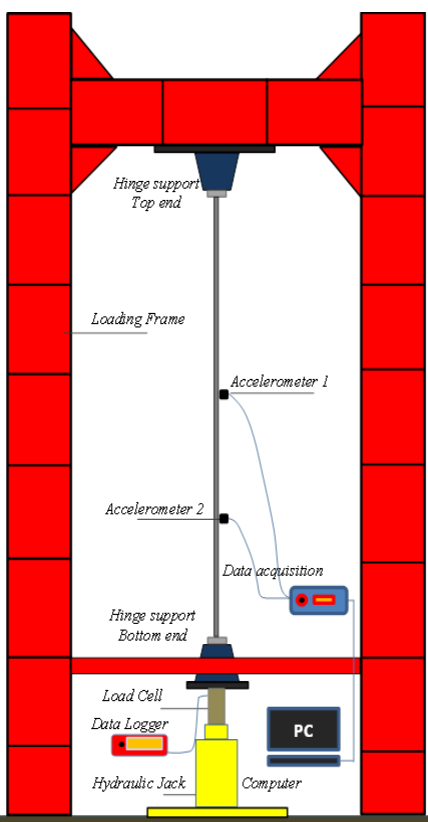

Fig. 7 - Set up of experimental testing 
The circular steel specimen was hanged in the vertical direction and pinned supported at both ends. The axial tensile load was applied using hydraulic jack then recorded by using load cell that placed beneath the bottom pin support. The data logger viewed the axial tensile force. The natural frequency of the specimen was recorded by using two accelerometers attached laterally at the half and the quarter span. The type of the accelerometers is Bruel \& Kjaer Type 4370 plugged on A/D Converter of Dewe-43 at one ends and. The analog signal responses recorded by accelerometer sensor were transferred to A/D converter of Dewe-43, converted into digital signals with a sampling rate of $250 \mathrm{~Hz}$ and analyzed by using FFT program using software Dewesoft program which had been installed in a laptop computer.

\section{Result and Discussion}

\subsection{Experimental Result}

Investigating the natural frequency of the specimen, the signal, recorded by the accelerometer sensor, were processed from the analog to digital signals using A/D converter then the signals were analyzed using the FFT program. Data of each specimen were presented in the relationship between natural frequency and amplitude as shown in Fig. 8 Fig. 12. The first natural frequency of specimen was presented in Table 2.

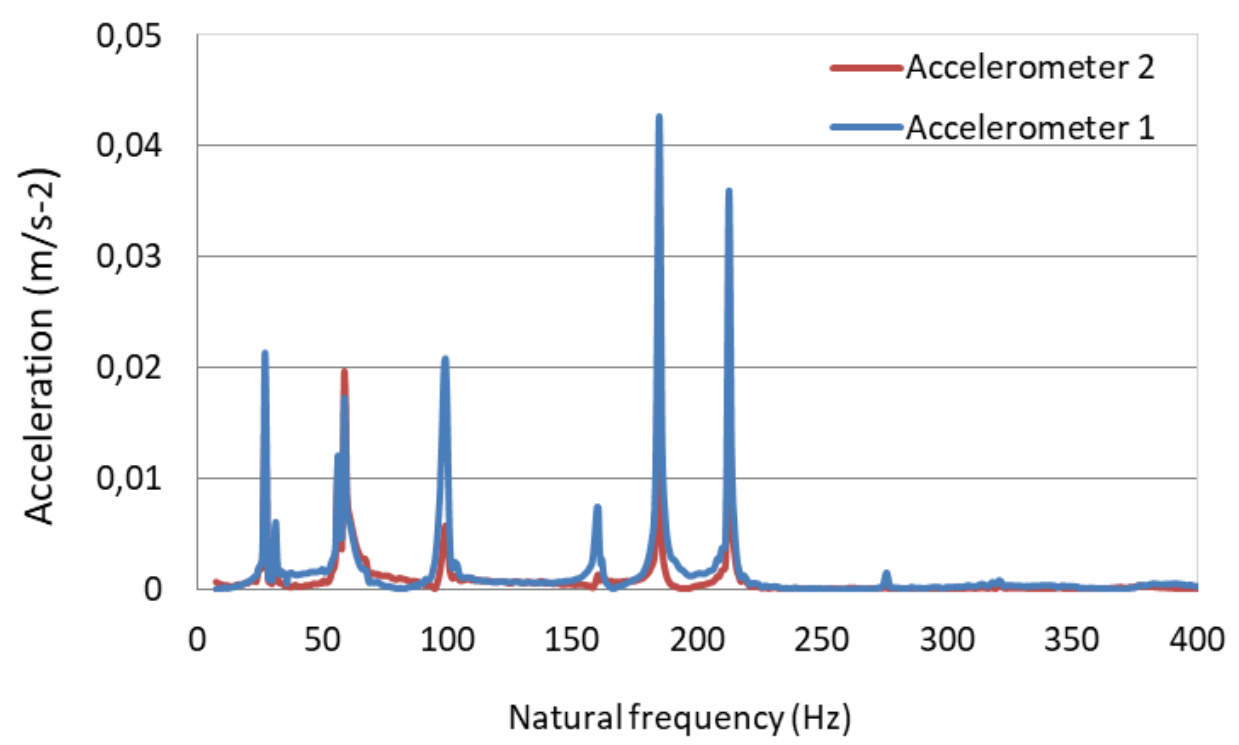

Fig. 8 - The natural frequency of the specimens using $\mathrm{I} / \mathrm{L}$ of 0.024

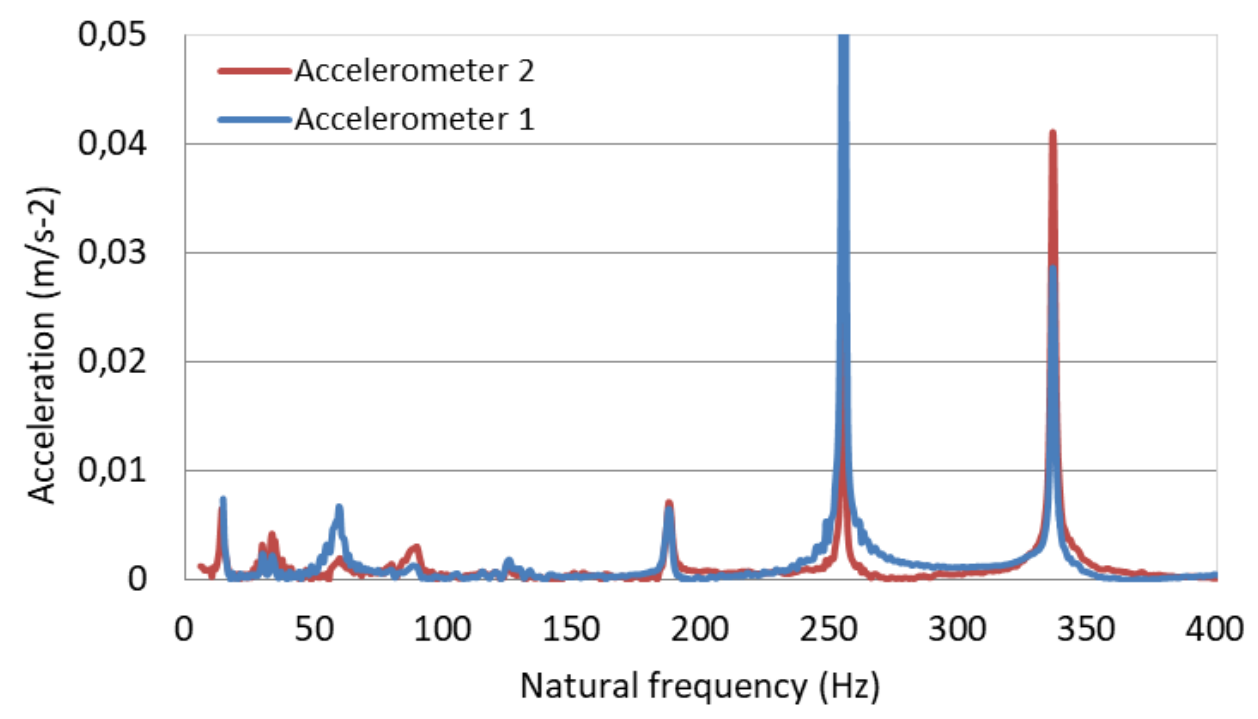

Fig. 9 - The natural frequencies of the specimens using $\mathrm{I} / \mathrm{L}$ of 0.058 


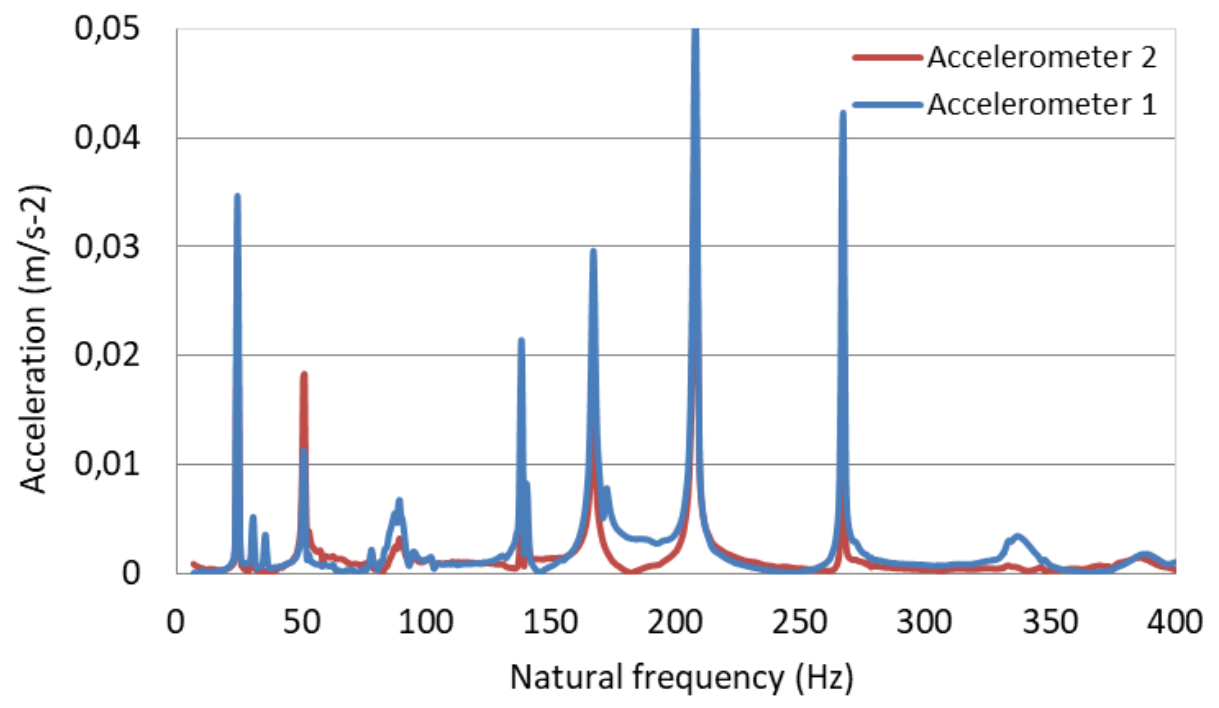

Fig. 10 - The natural frequencies of the specimens using $\mathrm{I} / \mathrm{L}$ of 0.082

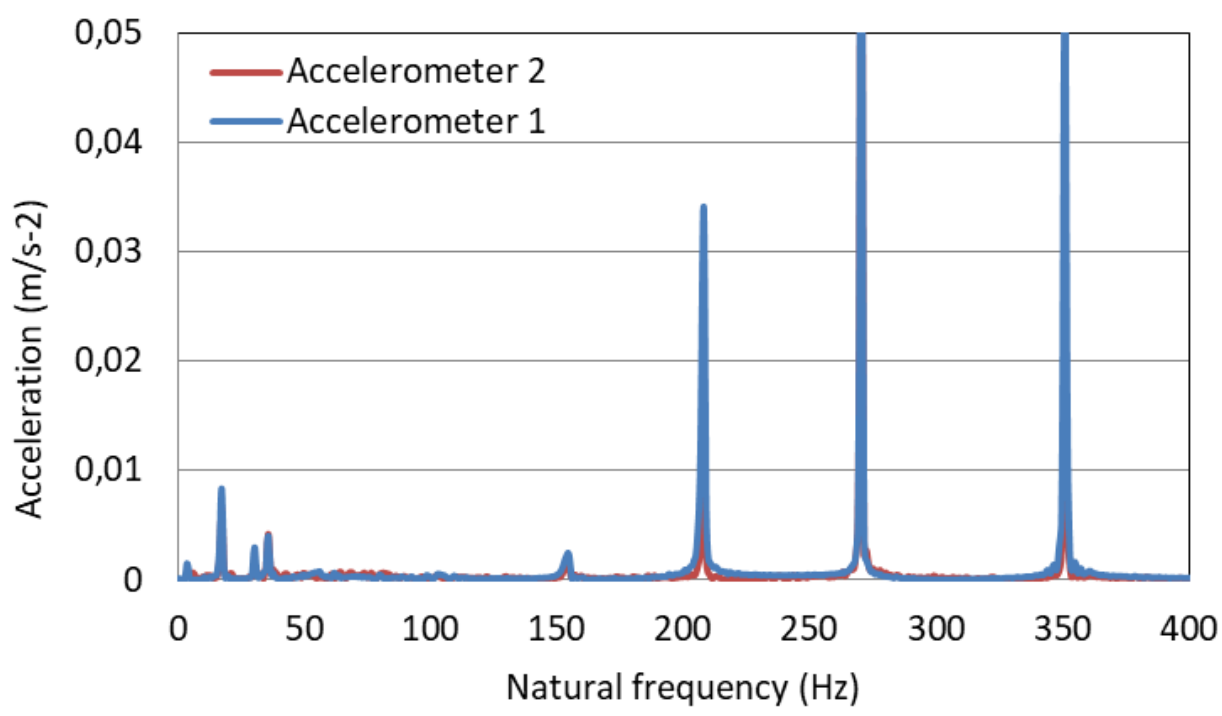

Fig. 11 - The natural frequencies of the specimens using $\mathrm{I} / \mathrm{L} \mathrm{of} 1.53$

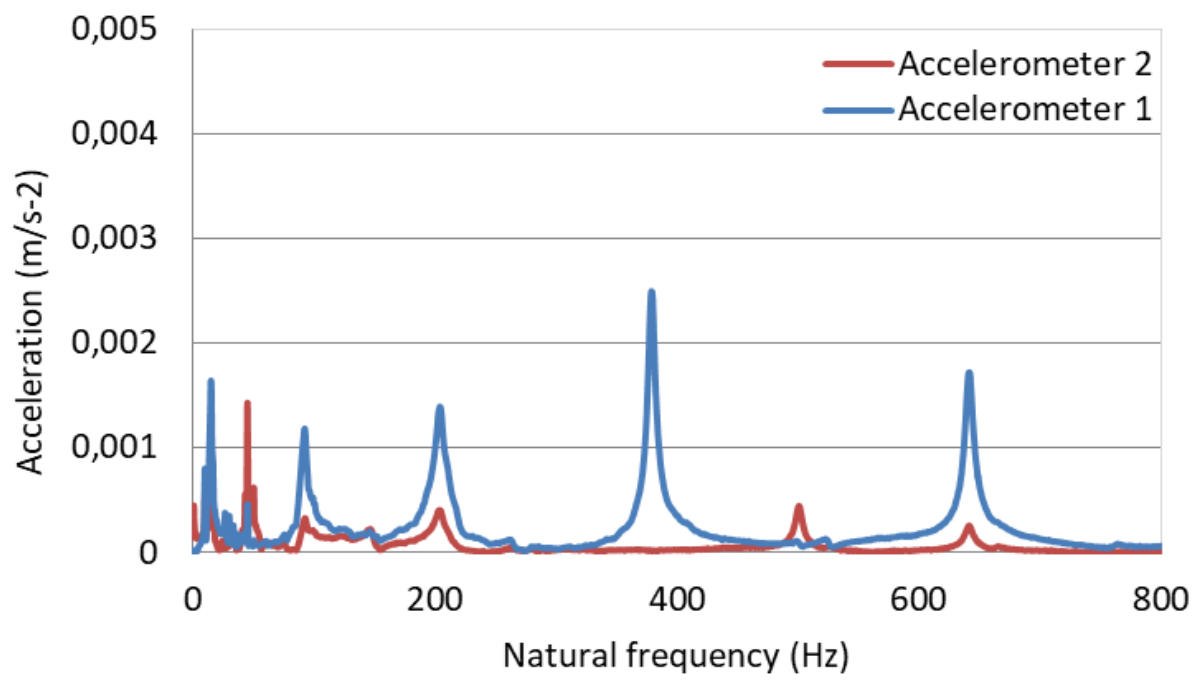

Fig. 12 - The natural frequencies of the specimens $I / L$ of 10.21 
Table 2 - First natural frequency of specimenas

\begin{tabular}{ccc}
\hline No & I/L & $\mathbf{f}_{\mathbf{n}}(\mathbf{H z})$ \\
\hline 1 & 0.024 & 32.28 \\
2 & 0.082 & 24.71 \\
3 & 0.580 & 17.90 \\
4 & 1.530 & 15.14 \\
5 & 10.210 & 14.65 \\
\hline
\end{tabular}

Fig 8-Fig 12 and Table 2 show that the specimen using a ratio of the moment of inertia to length (I/L) of 0.024 , $0.082,0.58,1.53$, and 10.21 have the first of natural frequency of $32.28 \mathrm{~Hz}, 24,71 \mathrm{~Hz}, 17,09 \mathrm{~Hz}, 15,14$ and $14,65 \mathrm{~Hz}$ respectively.

The higher the value of the ratio of I/L the greater the value of the diameter of the cross-section of the specimen could be. Increasing the diameter of the specimen will be folowed by the increasing of the bending stiffness (EI) as well as the mass per unit length $(\mathrm{m})$. In the analytical formula of beam-string theory, the natural frequency has directly proportional to the bending stiffness (EI) but has inversely proportional to the mass per unit length (m). Although the value of bending stiffness increases coincide with the value of mass per unit length, the natual frequency of specimens decreases. It shows that the influence of mass per unit length to the natural frequency was dominant than that of the bending stiffness.

\subsection{Numerical Result}

Numerical modeling, using specimen in Table 3, was simulated using Abaqus software. The first natural frequency was used as presented in Table 3.

Table 3 - Natural Frequency using Numerical Method

\begin{tabular}{ccc}
\hline No & I/L & Natural frequency (Hz) \\
\hline 1 & 0.024 & 34.97 \\
2 & 0.082 & 26.10 \\
3 & 0.580 & 17.50 \\
4 & 1.530 & 14.97 \\
5 & 10.210 & 14.68 \\
\hline
\end{tabular}

\subsection{Analytical Result}

Analytical method was conducted by using string theory and beam theory as presented in Eq.3 and Eq.23. The first natural frequency of specimens was shown in Table 4.

Table 4 - Natural frequency using analytical method

\begin{tabular}{cccc}
\hline No & I/L & String Theory (Hz) & Beam-string Theory (Hz) \\
\hline 1 & 0.024 & 34.71 & 34.83 \\
2 & 0.082 & 26.49 & 26.68 \\
3 & 0.580 & 16,24 & 17.06 \\
4 & 1.530 & 12.74 & 14.96 \\
5 & 10.210 & 7.87 & 14.88 \\
\hline
\end{tabular}

\subsection{Comparison between experimental, analytical and numerical}

The relationship between natural frequency and various parameters of I/L was shown in Fig 13. The diferencies of experimental, analytical and numerical results of the first pick of natural frequency of specimens (using ratio I/L of $0.024,0.082,0.58,1.53$, and 10.21) were presented in Table 5. 
Table 5 - The differencies natural frequency between analytical and numerical to experimental

\begin{tabular}{ccccc}
\hline No & I/L & \multicolumn{3}{c}{ Differencies natural frequency (\%) } \\
& & Analytical String Theory & Analytical Beam Theory & Numerical \\
Abaqus \\
\hline 1 & 0.024 & 6.27 & 6.51 & 6.20 \\
2 & 0.082 & 7.20 & 7.97 & 6.47 \\
3 & 0.580 & 9.27 & 6.48 & 5.14 \\
4 & 1.530 & 14.85 & 1.18 & 3.07 \\
5 & 10.210 & 46.27 & 1.59 & 1.29 \\
\hline
\end{tabular}

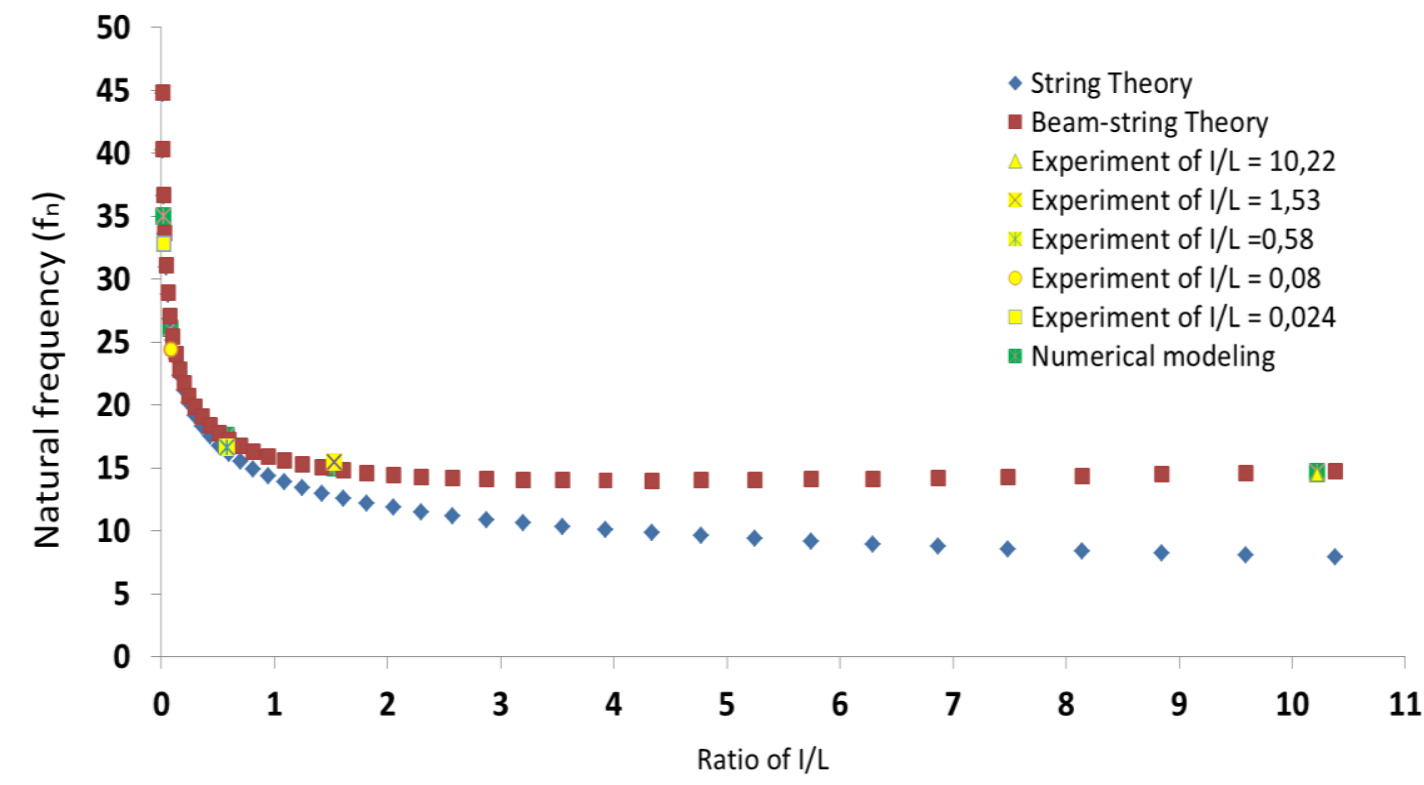

Fig. 13 - The comparison of natural frequency between experimental, numerical and analytical formula (using string and beam-string formula) in diference ratio of $I / L$

As shown in Table 5, natural frequency of experimental testing of the specimen using the ratio of I/L 0,024 has the diference of $6.27 \%$ to the analitical formulasion string theory, meanwile it has the diference of $6.51 \%$ to the analitical formulasion of beam-string theory and has the diference of $6.20 \%$ to the numerical modeling. The natural frequency of experimental testing of the specimen using the ratio of $\mathrm{I} / \mathrm{L} 0,082$ has the diference of $7.20 \%$ to the analitical formulasion string theory, meanwile it has the diference of $7.97 \%$ to the analitical formulasion of beam-string theory and has the diference of $6.47 \%$ to the numerical modeling.

The natural frequency of experimental testing of the specimen using the ratio of $\mathrm{I} / \mathrm{L} 0.58$ has the diference of $9.27 \%$ to the analitical formulasion string theory, meanwile it has the diference of $6.48 \%$ to the analitical formulasion of beam-string theory and has the diference of $5.14 \%$ to the numerical modeling. The natural frequency of experimental testing of the specimen using the ratio of $\mathrm{I} / \mathrm{L} 1.53$ has the diference of $14.85 \%$ to the analitical formulasion string theory, meanwile it has the diference of $1.16 \%$ to the analitical formulasion of beam-string theory and has the diference of $3.07 \%$ to the numerical modeling. The natural frequency of experimental testing of the specimen using the ratio of $\mathrm{I} / \mathrm{L} 10.21$ has the diference of $46.27 \%$ to the analitical formulasion string theory, meanwile it has the diference of $1.59 \%$ to the analitical formulasion of beam-string theory and has the diference of $1.29 \%$ to the numerical modeling.

Acording to beam-string theory in Eq 25, the parameters influencing the naural frequency of beam were the length, load, mode number, mass per unit langth and bending stifness. The specimens used in this research have the similar tof he boundary condition, length, mode number, axal load but it have the diference of mas per unit length and bending stifness. It mean that the natural frequency of secimens only influenced by the parameter of mass per unit length and bending stifness. For the specimen using I/L of 0.024 and 0.082 , have the diferencies of natural frequency between string formula and beam-string formula of $1.17 \%$ and $1.46 \%$ respectively.

On the other hand, specimen using $\mathrm{I} / \mathrm{L}$ of $0.58,1.53$ and 10.21 have the diferencies of natural frequency between string formula and beam-string formula of $2.79 \%, 13.67 \%$ and $44,68 \%$ respectively. The bigger the rasio of I/L of specimens the bigger the diferences between the natural frequency calculated by using analitical string and beam-string theory would be. The natural frequencies of experimental testing of specimens using ratio $\mathrm{I} / \mathrm{L}$ more than 0.082 are close 
to the analytical formula using beam-string theory. the effect of the bending stifness clearly raises on the specimen having the ratio of $\mathrm{I} / \mathrm{L}$ more than 0,082 . It can be concluded that the specimen using the ratio of I/L more than 0,082 , are cloas to the beam-string theory. On the other hand, the natural frequency of specimen using ratio of I/L lower than 0,082 , are cloas to thenatural frequency calculated by string theory as well as beam-string theory. It can be concluded that the effect of bending stifness can be neglected when the specimen having the ratio of I/L lower than 0,082 .

\section{Conclusion}

The objective of this research is to introduce the parameter of I/L to determine the apropriate of analitical formula between string and beam-string formula. The primary research was conducted by using several specimen varioused in diferences parameter of $\mathrm{I} / \mathrm{L}$. The result show that the effect of bending stifness can be neglected when the specimen having the ratio of $\mathrm{I} / \mathrm{L}$ lower than 0,082 . It can be concluded, that the analytical formula of string and beam-string theory can be used to estimate the axial tension force if the ratio of $\mathrm{I} / \mathrm{L}$ are lower than 0,082 . On the other hand, the analytical formula of beam-string theory can be used if the ratio of I/L more than 0.082 .

\section{Acknowledgement}

Author's would like to thank you to Universitas Muhammadiyah Yogyakarta, Indonesia.

\section{References}

Clough, R.W \& Penzien, J. (1995). Dynamic of Structure Computer and Structure Inc., Berkeley, CA 94704, USA: University Ave

Fang, Z., \& Wang. J. (2012). Practical Formula for Cable Tension Estimation by Vibration Method. Journal of Bridge Engineering, 17(1), 161-164

Irvine, H. M. (1981). Cable Structure, Cambridge, M.A, USA.: MIT Pers

Kim, B. H., \& Park, T. (2007). Estimation of cable tension force using the frequency-based system identification method. Journal of Sound Vibration, 204(3-5), 660-676

Nugroho, G., Priyosulistyo. H., \& Suhendro, B. (2014). Evaluation of tension force using vibration technique related to string and beam theory to ratio of moment of inertia to span. Procedia Engineering Volume 95, $225-230$

Russell, J. C., \& Lardner, T. J. (1998). Experimental determination of frequencies and tension for elastic cables. Journal of Engineering Mechanis. Volume 124(10), 1067-1072

Saxon \& Chan. (1953). Mode of vibration of a suspended chain. Quarterly Journal of Mechanics and Applied Mathemathics, 6 (3) ,273-285

Soojin, C., Jinsuk, Y., Sung, W.S., Hyung J. J., Chung, B.Y., \& Ming, L.W. (2013). Comparative Field Study of Cable Tension Measurement for a Cable-Stayed Bridge. Journal of Bridge Engineering, 18(8), 748-757

Tabatabai, H., Mehrabi, A. B., \& Yen, W. P. (2003). Bridge stay cable condition assessment using vibration measurement techniques. Proc. SPIE Int. Soc. Opt. Eng. 3400, 194-204

Zui, H., Shinke, T., \& Namita, Y. (1998). Practical formulas for estimation of cable tension by vibration method. Journal of Structure Engineering, volume 122(6), 651-656 